ISSN 1870-4069

\title{
Exploring Digital and Manual Modalities in Educational Activities for Children with ADHD
}

\author{
Raymundo Cornejo ${ }^{1}$, Fernando Martinez ${ }^{2}$ \\ ${ }^{1}$ CONACYT, Universidad Autónoma de Chihuahua, \\ Chihuahua, Mexico \\ ${ }^{2}$ Facultad de Ingeniería, Universidad Autónoma de Chihuahua, \\ Chihuahua, Mexico \\ rcornejo@conacyt.mx,fmartine@uach.mx
}

\begin{abstract}
Planning and conducting educational activities for children with ADHD represents a methodical and precise endeavor. Teachers plan the educational activities but also have to adapt each educational session based on the child's emotional state. Digital technologies are often suggested as a means to provide engaging learning resources and to help children with their academic achievements. Therefore, we developed a supportive software based on an educational activity where we aim to help children reinforce learning abilities. We conducted a controlled study to compare the manual and digital modalities of the educational activity. Our analysis reveals sentiment findings when new technology is introduced. Our findings suggest that using educational activities enhanced with digital technology has potential benefits and may allow teachers and children achieve academic goals yet providing a ludic educational experience for children.
\end{abstract}

Keywords: Ludic experiences, affective states, attention deficit disorder, learning difficulties.

\section{Introduction}

Attention deficit hyperactivity disorder (ADHD) is the most common neurobehavioral disorder diagnosed in U.S. children. In 2011-2013, 13.3\% of boys and 5.6\% of girls aged 4-17 had ever been diagnosed with ADHD [1]. Prior research [2,3] have found similar prevalence percentages worldwide suggesting geographic location plays a limited role in ADHD prevalence. Particularly, the Mexican Psychiatric Services Office estimates 3.5\% of Mexican children have been diagnosed with ADHD [4].

Children diagnosed with ADHD experience a wide range of challenges with daily life and their education, compromising the expectations from parents and teachers according to skills presented in neurotypical children of the same age [5]. For instance, they face problems identifying individual graphemes or words while constructing meaningful sentences related to reading assignment. Furthermore, short term memory 
issues may arise and contribute to dyslexia or dysgraphia diagnosis [6], leading to low average grades, failed grades, expulsions, or increased school dropout rates. Along with learning challenges these children have issues while interacting with others peers due to inattention to what is happening around them or what others are saying [7]. Therefore, there seems to be a need to support the efforts teachers have to do to implement strategies and approaches to engage children in educational and social activities.

Digital technologies have been suggested as a means to provide creative, intuitive and engaging learning experiences, which can help teachers better planning their students' educational goals [8]. However, designing technological yet instructional content for children with ADHD is challenging. In this paper we present the findings of an initial technological approach, where children are encourage to play with digitaleducational resources. We argue that with our digital instructional resource, children can practice memory, attention and reasoning skills while the activities' design and technology keeps them engaged. Furthermore, the design and implementation of these kinds of technologies might be an alternative to physicians who often rely on pharmacological interventions as initial and/or sole treatment for children with ADHD [9].

\section{Related Work}

Digital technologies are conceived as a vehicle to deliver instructional educational content [10]. Especially during early childhood, playing positively influences important psychological, sociological, and intellectual developments. For instance, by means of video games researchers have studied how seven-years-old children make connections around mythology assignments. Users confront problems and make use of their expertise to solve puzzles before moving to the next challenge, and this engaging activity would never stops because of the ludic approach to deliver learning content [11]. Technology is also seen as a mediator for teaching cognitively demanding tasks. An example of these are educational tools that support the teaching of mathematics skills such as the manipulation of fraction equivalence [12].

The use of these and other technology-based instructional resources offer new options for children with ADHD. For instance, effective instruction might be reached using graphics, words and sounds, given that the recreation of real world scenarios is possible. Computers also allow repeated trials and let instructors organize content into smaller chunks of information [13]. The review offered by this author indicates that technology could create highly stimulating instructional environment, could envisage a promising approach for helping students gain self-control over their behaviors, or provide biofeedback training so that both students and therapists' are informed about brain activity.

Nevertheless one of the main symptoms exposed by children with ADHD is their short attention span, which leads to poor academic performance. For therapists the measuring of this neurobiological condition is commonly based on a subjective observational approach. Assistive technologies can help therapists make the inattentive 
behavior assessment easier given that current gaze tracking devices localize the focus of attention on a display. In [14] authors, explored the design of a system aiming to evaluate a gaze-based attentive user interface that monitors the child eyes. Such a system could be used to manage educational content based on the level of attention the child keeps with the instructional material. A more robust technology-based approach is proposed by [15] to detect divided attention in reading activities. While children were reading the environment was fueled with distractors in the format of multitasking instructions or environmental noise.

\section{Technological Solution}

During period of four months we conducted a case study using user centered design techniques to design and implement an educational software tool for children diagnosed with ADHD at the Instituto José David in Chihuahua, México. The software was designed as a ludic experience with educational tasks aimed to reinforce learning abilities such as: attention, cognition, memory, and visuospatial memory. The software, for example, uses iconic cards to stimulate short term memory. Cards are presented to the player during a few seconds and the child has to select the previously shown cards immediately after cards disappear. Cards are in an unordered and larger set of cards to evaluate short term memory (see Figure 1a). In addition, visuospatial memory is reinforced when children have to summarize and recall information about the spatial location of objects on a grid (see Figure 1b).

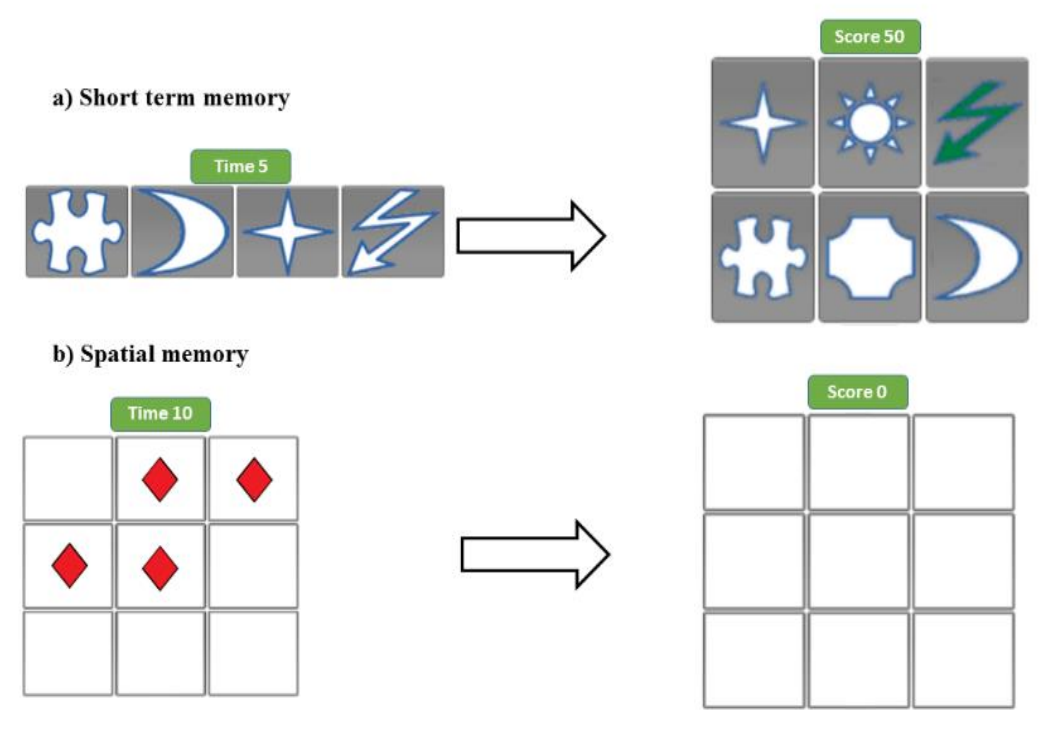

Fig. 1. Software's games: a) memory and b) visuospatial. 


\section{Methods and Evaluation}

Researchers conducted a controlled between subjects experiment to evaluate children's attention and frustration levels while conducting one short term memory activity. Participants $(\mathrm{N}=15)$ were students between 8 and 11 years old ( 6 female). Students were recruited from among ADHD patients at Instituto José David in Chihuahua, México and consent was obtained from their parents. Evaluation consisted in four phases: 1) playing session with a digital game modality, 2) answer a questionnaire about the game, 3) play with the manual modality, and 4) answer a questionnaire about the game.

Participants were taken to Gesell room and assigned to start either with the digital or physical version of the game. A therapist on language and learning explained to each participant the dynamics of the game and how to play it during each playing session. Based on the child's excitement level, each child was given a specific time (avg. 5 min) to calm down and understand how to play and short after the therapist initiated the game. The digital modality was played with the Leap Motion ${ }^{1}$ device and the manual modality was played with tangible game cards.

All playing sessions were video recorded with a webcam and a video camera and observed by a psychologist who analyzed each session independently. Furthermore, we used the Emotiv EEG device to track and generate real time reports on children affective state. EEG data was additionally analyzed in order to understand frustration (FM, FD), excitement (EM, ED), and engagement (EBM, EBD). Children were moved to a waiting area after each playing session and one researcher applied a questionnaire to assess each game modality.

The questionnaire evaluates user experience on attention and satisfaction for both manual and digital. It consisted on 9 questions for each modality and each question had a Likert scale with answers from "never" to "very frequently". Children played with both modalities of the educational activity $(\mathrm{N}$ Sessions $=60$, Total of Questionnaires $=$ 60). Questionnaire's data was analyzed using median values to understand satisfaction and attention levels between manual (AM) and digital (AD) modalities. We conducted Wilcoxon signed-rank test due to the non-normal distribution of data.

Table 1. Wilcoxon signed rank test of FD and FM.

\begin{tabular}{|c|c|c|c|c|}
\hline & & $\mathbf{N}$ & Mean Rank & Sum of Ranks \\
\hline \multirow[t]{4}{*}{$F D-F M$} & Negative Ranks & $1^{\mathrm{a}}$ & 2.50 & 2.50 \\
\hline & Positive Ranks & $11^{\mathrm{b}}$ & 6.86 & 75.50 \\
\hline & Ties & $3^{c}$ & & \\
\hline & Total & 15 & & \\
\hline$Z$ & $-2.915^{\mathrm{d}}$ & & & \\
\hline Asymp. Sig. (2-tailed) & .004 & & & \\
\hline
\end{tabular}
a. $F D<F M ; b . F D>F M ; c . F D=F M$; d. Base on negative ranks

1 https://www.leapmotion.com/ 


\section{$5 \quad$ Findings}

Developing educational tools entails aspects from supporting teachers to creating engaging, yet educational, activities for children. In this section we present an overview of how current technology can be used for educational purposes based on sentiments.

\subsection{Sentiment Analysis}

During our study, children with ADHD showed important levels of frustration while playing the educational game in digital mode. Our findings indicate notorious differences on medians of frustration levels on both modalities. The Leap Motion modality has a higher frustration median than the manual activity (FM=2.0 and $\mathrm{FD}=4.0$ ). This difference is further validated with the Wilcoxon signed rank test that indicates to what extent frustration levels with the Leap Motion interaction are statistically significant higher than the frustration levels with the manual activity (see Table $1, \mathrm{Z}=-2.915, \mathrm{p}<0.004$ ). To our knowledge, frustration has not been related with learning performance. Nevertheless, it may leads to children unwillingness to perform the educational activity. Frustration might arise due to the novelty of technology. During our study, this was the first time children used the Leap Motion technology and at times the Leap Motion did not perform as children would had expected, thus creating these significant levels of frustration. Even with these levels of frustration, we observed how there is also a significant difference between the digital (ED) and manual (EM) excitement levels (Medians: $\mathrm{ED}=4.0, \mathrm{EM}=2.0$ ). The Wilcoxon signed rank test indicates to what extent excitement levels are significantly higher with the Leap Motion technology than excitement levels with the manual activity (see Table $2, Z=-2.004, p$ $<0.045)$. This entails an interesting scenario where children feel frustrated with the new digital learning activities but also are more excited and motivated to complete them.

Table 2. Wilcoxon signed rank test of ED and EM.

\begin{tabular}{c|c|c|c|c|}
\hline \multirow{4}{*}{$E \boldsymbol{E D}-\boldsymbol{E M}$} & Negative Ranks & $4^{\mathrm{a}}$ & 3.5 & 14.00 \\
\cline { 2 - 5 } & Positive Ranks & $8^{\mathrm{b}}$ & 8.00 & 64.00 \\
\hline & Ties & $3^{\mathrm{c}}$ & & \\
\cline { 2 - 5 }$Z$ & Total & 15 & & \\
\cline { 2 - 5 }$Z$ & $-2.004^{\mathrm{d}}$ & & & \\
\hline Asymp. Sig. (2-tailed) & .045 & & & \\
\hline
\end{tabular}

a. $E D<F M ; b . E D>E M ; c . E D=E M$; d. Base on negative ranks

In this regard, we believe that children might adapt to the technology interaction mode over time and with dynamic learning activities we might sustain excitement levels. Therefore, we can expect lower levels of frustration across a longitudinal study. In sum, a digital version with ludic excitement interactions might be the best option for educational and digital processes. 


\subsection{Digital Modality or Manual Preference}

Although children presented significant levels of excitement over digital learning activity, we did not find a clear preference over the manual modality. Analyzing satisfaction data we observed how median satisfaction for manual activity was 5.0 as it was for the median satisfaction for the digital activity. We can infer that ludic learning activities provide a positive affective state since both modalities had the highest satisfaction level from our survey. In addition, both ludic modalities present high median attention level $(\mathrm{AD}=5.0, \mathrm{AM}=5.0)$. Nonetheless children diagnosed with ADHD are characterized with inattention during learning activities, our participants were satisfied while playing both modalities and were attentive to the dynamics of the game despite the frustration levels due to digital technology. Median levels for the engagement state show how children were enthusiastic players $(\mathrm{EBD}=4.0, \mathrm{EBM}=$ 4.0) with few instances of boredom while playing. Regardless of these sentiment findings, there is not a clear children's preference over one modality of the learning activity. Further research is needed to monitor attention and satisfaction levels across time and exploit the benefits of using game-based learning technologies to promote excitement while learning.

\section{Discussion}

While prior work has highlighted the importance of technologically enriching classrooms or learning therapy sessions, our analysis focuses on identifying opportunities to support ludic learning activities with emergent technology. Technology provides important information around children's sentiments that can be used to enrich the learning experience. Nonetheless, abstract concepts such as attention can be difficult to monitor or infer. Therefore there is the opportunity to design and develop robust solutions to create intelligent learning management systems supported by sentiment analysis. In the next sections, we describe current limitations as opportunities of improvement but also alternative technologies to create more robust solutions.

\subsection{EEG Limitations \& Complementary Technologies}

Teachers plan ahead the learning activities according the children' ADHD diagnosis but also make adjustments on daily basis based on the emotional state of each child. Adapting the learning activities might be troublesome since teachers might not be exactly certain how the emotional state of the child is. Teachers have to react when they observe the child presents a negative attitude (e.g. inattention). Throughout our analysis we observed how current EEG technology allows to analyze and determine the emotional state of the child while conducting certain educational activity. Providing this information to teachers in real time might alleviate or reassure the emotional assessment conducted during therapy or educational sessions. Nonetheless, current mobile EEG technology can be somewhat invasive or inaccurate for complex cognitive processes (e.g. attention). During our study, children were asked to wear a headset in 
order to conduct our EEG data gathering, this is somewhat intrusive and not transparent for the children with the risk of inducing the sentiment of feeling observed or evaluated. Furthermore, identifying complex cognitive processes with the EEG data was challenging since identifying the sensory brain signals generated by such processes is still an open problem. Even for experienced psychologists this represents a challenging endeavor, for example, the psychologist involved in our participants' sessions stated how difficult is to determine if children were attentive to the learning activity or just their eyes were fixed on the activity but not paying attention to it. Therefore, current educational activities might benefit from incorporating complementary technologies to enhance accuracy detection of emotional states. Biometrics technology (e.g. eyetracking or facial recognition) can be introduced to supplement emotional information and help detect when children are frustrated or are losing focus on the educational activity. Providing robust and less invasive mechanisms to infer emotional states on children have the potential benefits of conducting meaningful educational activities where children diagnosed with ADHD can be engaged and attentive.

\subsection{Tradeoffs of New Technology vs ADHD}

Children diagnosed with ADHD have been socially considered vulnerable since they might be subject to disadvantages compared with neurotypical children. In our study we decided to focus our efforts to empower and support learning activities for this population. Our findings showed higher frustration levels when using technology than those with the manual activity. Although these preliminary findings might suggest manual activities should be preferred over a digital one, we argue that these frustration levels were due to the novelty of the technology, and once the novelty wears off children will get accustomed to interact with such interfaces. Nevertheless, enriching learning environments with technology also conveys additional benefits to improve educational sessions. First, our findings illustrate how children experienced significant levels of excitement while using the digital modality of the educational activity. This experienced enjoyment alleviates the teacher's concerns of keeping children engaged on the learning activity and motivates students to keep assisting to the Institute. Furthermore, through sentiment analysis, intelligent learning management systems can be implemented to adequate the learning activity based on the emotional state of each child. For example, our system can log the emotional state over time and adapt dynamically the activity based on the emotions to keep the child engaged with the memory activity. Future learning management systems might also have a user input to avoid uncertainty when the teacher can disagree with the evaluation made by the system and input new values based on their expertise. Even with the high levels of frustration, we believe future learning systems should take into account the integration of different mechanisms to identify emotional state of the child and dynamically adequate digital learning activities which could help alleviate the teachers' burden.

\section{Conclusion}

Planning and executing educational activities for children with ADHD is a challenging endeavor. Developing ludic and digital tools to support teachers on their daily sessions 
uncovers new challenges but also provides benefits. We acknowledge the length of the study as a limitation; nevertheless our findings suggest potential benefits of enriching educational activities with technology. Future learning systems can adapt educational activities with machine learning algorithms by analyzing emotional states to reduce the levels of frustration and maintain children engaged with the activities. Nevertheless, teachers are employing ludic activities to instruct children diagnosed with ADHD.

\section{References}

1. Pastor, P., Reuben, C., Duran, C., Hawkins, L.: Association between diagnosed ADHD and selected characteristics among children aged 4-17 years: United States, 2011-2013. NCHS Data Brief, 201 (2015)

2. Thomas, R., Sanders, S., Doust, J., Beller, E., Glasziou, P.: Prevalence of AttentionDeficit/Hyperactivity Disorder: A Systematic Review and Meta-analysis. Pediatrics, (2015)

3. Polanczyk, G., de Lima, M. S., Horta, B. L., Biederman, J., Rohde, L. A.: The worldwide prevalence of ADHD: a systematic review and metaregression analysis. Am. J. Psychiatry, 164, pp. 942-948 (2007)

4. Posa-Diaz, M. del P., Valenzuela-Moreno, M. V., Becerra-Alcantara, D.: Guía clínica: Transtorno por déficit de atención e hiperactividad

5. Romero-Ayuso, D. M., Maestu, F., Gonzalez-Marques, J., Romo-Barrientos, C., Andrade, J. M.: Executive dysfunction in attention deficit hyperactivity disorder in childhood. Rev. Neurol, 42, pp. 265-271 (2006)

6. Pineda, D. A.: La función ejecutiva y sus trastornos. Rev. Neurol, 30, pp. 764-768 (2000)

7. Barkley, R. A.: Attention-deficit/hyperactivity disorder. In: Child psychopathology, pp. 75-143 (2003)

8. Baker, R. S., Corbett, A. T., Koedinger, K. R., Wagner, A. Z.: Off-task behavior in the cognitive tutor classroom. In: Proceedings of the 2004 conference on Human factors in computing systems, CHI '04, ACM Press, New York, New York, USA, pp. 383-390 (2004)

9. Zasler, N. D., Martelli, M. F., Jacobs, H. E.: Chapter 32 - Neurobehavioral disorders. In: Neurology, Neurological Rehabilitation, Elsevier, pp. 377-388 (2013)

10. Amory, A.: Game object model version II: a theoretical framework for educational game development. Educ. Technol., Res. Dev., 55, pp. 51-77 (2007)

11. Gee, J. P.: What Video Game Have to Teach Us About Learning and Literacy. Comput. Entertain., 1, pp. 1-4 (2003)

12. Kong, S. C., Kwok, L. F.: A cognitive tool for teaching the addition/subtraction of common fractions: a model of affordances. Comput. Educ., 45, pp. 245-265 (2005)

13. Xu, C., Reid, R., Steckelberg, A.: Technology Applications for Children with ADHD: Assessing the Empirical Support. Educ. Treat., Child, 25, pp. 224-248 (2002)

14. Asiry, O., Shen, H., Calder, P.: Extending Attention Span of ADHD Children through an Eye Tracker Directed Adaptive User Interface. In: Proceedings of the ASWEC 2015 24th Australasian Software Engineering Conference on - ASWEC' 15, ACM Press, New York, New York, USA, Vol.II, pp. 149-152 (2015)

15. Rodrigue, M., Son, J., Giesbrecht, B., Turk, M., Höllerer, T.: Spatio-Temporal Detection of Divided Attention in Reading Applications Using EEG and Eye Tracking. In: Proceedings of the 20th International Conference on Intelligent User Interfaces - IUI '15, ACM Press, New York, New York, USA, pp. 121-125 (2015) 